\title{
Finite-Element Theory of Transport in Ferromagnet-Normal Metal Systems
}

\author{
Arne Brataas,* Yu. V. Nazarov, and Gerrit E. W. Bauer \\ Department of Applied Physics and Delft Institute of Microelectronics and Submicrontechnology, Delft University of Technology, \\ Lorentzweg 1, 2628 CJ Delft, The Netherlands
}

(Received 4 June 1999)

\begin{abstract}
We formulate a theory of spin dependent transport in an electronic circuit involving ferromagnetic elements with noncollinear magnetization which is based on the conservation of spin and charge current. The theory considerably simplifies the calculation of the transport properties of complicated ferromagnet-normal metal systems. We illustrate the theory by considering a novel three-terminal device.
\end{abstract}

PACS numbers: 72.10.Bg, 75.70.Pa

Electron transport in hybrid systems involving ferromagnetic and normal metals has been shown to exhibit new phenomena due to the interplay between spin and charge. The giant magnetoresistance (GMR) effect in metallic magnetic multilayers is a result of spin dependent scattering [1]. The manganese oxides exhibit a colossal magnetoresistance [2] due to a ferromagnetic phase transition. The dependence of the current on the relative angle between the magnetization directions has been reported in transport through tunnel junctions between ferromagnetic reservoirs [3]. Transport involving ferromagnets with noncollinear magnetizations has also been studied theoretically in Ref. [4]

Johnson and Silsbee demonstrated that spin dependent effects are also important in systems with more than two terminals [5]. Their ferromagnetic-normal-ferromagnetic $(F-N-F)$ device manifests a transistor effect that depends on the relative orientation of the magnetization directions. Recently, another three-terminal spin electronics device was realized; a ferromagnetic single-electron transistor [6]. In this case the current depends on the relative orientation of the magnetization of the source, the island and the drain, and of the electrostatic potential of the island tuned by a gate voltage [7].

These examples illustrate that devices with ferromagnetic order deserve a thorough theoretical investigation. Inspired by the circuit theory of Andreev reflection [8], we present a finite-element theory for transport in hybrid ferromagnetic-normal metal systems based on the conservation of charge and spin current. We demonstrate that spin transport can be understood in terms of four generalized conductances for each contact between a ferromagnet and a normal metal. The relations between these conductance parameters and the microscopic details of the contacts are derived and calculated for diffuse, tunnel, and ballistic contacts. Finally, we illustrate the theory by computing the current through a novel three-terminal device.

Let us first explain the basic idea of the finite-element theory of spin transport. The system can be divided into (normal or ferromagnetic) "nodes," where each node is characterized by the appropriate generalization of the distribution function, viz. a $2 \times 2$ distribution matrix in spin space. The nodes are connected to each other and to the reservoirs by "contacts" which limit the total conductance but are arbitrary otherwise. The charge and spin current through the contacts is related to the distribution matrices of the adjacent nodes. Provided these relations are known, we can solve for the $2 \times 2$ distribution matrices in the nodes under the constraint of conservation of spin and charge current in each node and thus determine the transport properties of the system. These macroscopic relations for each contact can be found in terms of the microscopic scattering matrices in the spirit of the Landauer-Büttiker formalism [9]. The scattering matrices can be calculated using different models such as a two-spin band model or realistic band structures and for various contacts, e.g., ballistic or diffuse wires or tunnel junctions. Phase coherent scattering as in resonant tunneling devices and effects like the Coulomb blockade can be included, in principle, by calling the double barrier a "contact" with complex scattering properties, but these complications will be disregarded in the following.

The device depicted in Fig. 1 will serve to illustrate our approach. Several contacts attach a normal metal node to (ferromagnetic or normal) metallic reservoirs. We assume that the resistances of the contacts are much larger than the resistance of the node. This is fulfilled when the area of the contact is sufficiently smaller than the cross section of the node or when the contacts are in the tunneling regime. The current through the system and the distribution matrix in the node are determined by the properties of the contacts. The reservoirs are supposed to be large and in local equilibrium with a chemical potential $\mu_{\alpha}$, where the subscript $\alpha$ labels the reservoirs. The energy dependent distribution matrix in the (ferromagnetic) reservoir is then diagonal in spin space $\hat{f}_{\alpha}^{F}(\epsilon)=\hat{1} f\left(\epsilon, \mu_{\alpha}\right)$, where the caret denotes a $2 \times 2$ matrix in spin space, $\hat{1}$ is the unit matrix, and $f\left(\epsilon, \mu_{\alpha}\right)$ is the Fermi-Dirac distribution function. The direction of the magnetization is denoted by the unit vector $\mathbf{m}_{\alpha}$. When the chemical potentials of the reservoirs are not identical, the normal metal node is not in equilibrium, and there can be a spin accumulation on the normal metal node. The distribution is therefore represented by a $2 \times 2$ matrix in spin space $\hat{f}^{N}(\epsilon)$, which allows a spin 


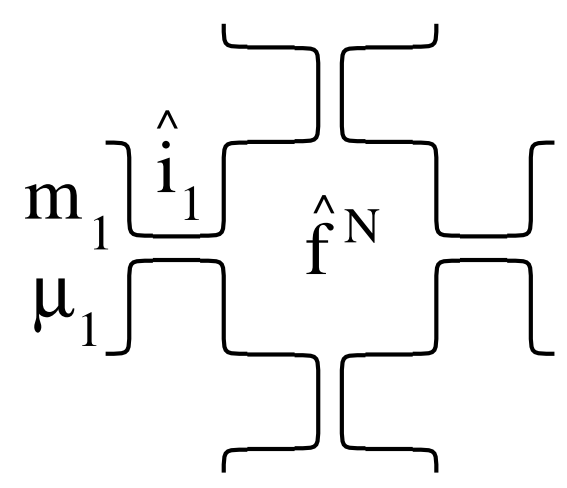

FIG. 1. A normal node connected to ferromagnetic reservoirs characterized by the chemical potentials $\mu_{\alpha}$ and the magnetization vector $\mathbf{m}_{\alpha}$. The distribution matrix in the normal node $\hat{f}^{N}$ can be found from the Kirchoff rules for the spin currents $\hat{i}_{\alpha}$.

accumulation with arbitrary direction of the spins. The normal metal node is considered to be large and chaotic either because of impurity scattering inside the node or because of scattering at irregularities of its boundary. The distribution matrix inside the node is therefore isotropic in momentum space and depends only on the energy of the particle.

The current through a contact is determined by its scattering matrix, the Fermi-Dirac distribution function of the adjacent ferromagnetic reservoir and the $2 \times 2$ nonequilibrium distribution matrix in the normal node. The current is evaluated close to the contact on the normal side. The $2 \times 2$ current in spin space per energy interval at energy $\epsilon$ leaving the node is

$$
\begin{aligned}
\frac{h}{e^{2}} \hat{i}= & \sum_{n m}\left[\hat{r}^{\mathrm{nm}} \hat{f}^{N}\left(\hat{r}^{\mathrm{nm}}\right)^{*}+\hat{t}^{\mathrm{nm}} \hat{f}^{F}\left(\hat{t}^{\mathrm{nm}}\right)^{*}\right] \\
& -M \hat{f}^{N},
\end{aligned}
$$

where $M$ is the number of propagating channels, $\hat{r}^{n m}(\epsilon)$ is the reflection matrix for an electron coming from the normal metal in mode $m$ being reflected to mode $n$, and $\hat{t}^{n m}(\epsilon)$ is the transmission matrix for an electron from the ferromagnet in mode $m$ transmitted to the normal metal in mode $n$. The total current is obtained by integrating over the energies, $\hat{I}=\int d \epsilon \hat{i}(\epsilon)$. The current in the contact is thus completely determined by the scattering matrix of the contact, and the distribution matrices.

The $2 \times 2$ nonequilibrium distribution matrix in the node in the stationary state is uniquely determined by current conservation

$$
\sum_{\alpha} \hat{i}_{\alpha}=\left(\frac{\partial \hat{f}^{N}}{\partial t}\right)_{\text {rel }}
$$

where $\alpha$ labels different contacts and the term on the right-hand side describes spin relaxation in the normal node. The right-hand side of Eq. (2) can be set to zero when the spin current in the node is conserved, i.e., when an electron spends much less time on the node than the spin-flip relaxation time $\tau_{\text {sf }}$. If the size of the node in the transport direction is smaller than the spin-flip diffusion length $l_{\mathrm{sf}}=\sqrt{D \tau_{\mathrm{sf}}}$, where $D$ is the diffusion coefficient, then the spin relaxation in the node can be introduced as $\left(\partial \hat{f}^{N} / \partial t\right)_{\text {rel }}=\left[\hat{1} \operatorname{Tr}\left(\hat{f}^{N}\right) / 2-\hat{f}^{N}\right] / \tau_{\text {sf }}$. If the size of the node in the transport direction is larger than $l_{\text {sf }}$ the simplest finite-element transport theory fails and we have to use a more complicated description with a spatially dependent spin distribution function [10]. Equation (2) gives the $2 \times 2$ distribution matrix of the node in terms of Fermi-Dirac distribution functions of the reservoirs. These distribution functions are determined by voltages of the reservoirs. Those voltages are either set by voltage sources or determined by conventional circuit theory.

We will now demonstrate that the relation (1) between the current and the distributions has a general parametrized form. Spin-flip processes in the contacts are disregarded, so that the reflection matrix for an incoming electron from the normal metal can be written as $\hat{r}^{n m}=$ $\sum_{s} \hat{u}^{s} r_{s}^{n m}$, where $s=\uparrow, \downarrow, r_{s}^{n m}$ are the spin dependent reflection coefficients in the basis where the spin quantization axis is parallel to the magnetization in the ferromagnet, $\hat{u}^{\dagger}=(\hat{1}+\hat{\boldsymbol{\sigma}} \cdot \mathbf{m}) / 2, \hat{u}^{\downarrow}=(\hat{1}-\hat{\boldsymbol{\sigma}} \cdot \mathbf{m}) / 2$, and $\hat{\boldsymbol{\sigma}}$ is a vector of Pauli matrices. Similarly, for the transmission matrix $\hat{t}^{n m}\left(\hat{t}^{n m}\right)^{*}=\sum_{s} \hat{u}^{s}\left|t_{s}^{n m}\right|^{2}$, where $t_{s}^{n m}$ are the spin dependent transmission coefficients. Using the unitarity of the scattering matrix, we find that the general form of the relation (1) reads

$$
\begin{aligned}
\hat{i}= & G^{\dagger} \hat{u}^{\dagger}\left(\hat{f}^{F}-\hat{f}^{N}\right) \hat{u}^{\uparrow}+G^{\downarrow} \hat{u}^{\downarrow}\left(\hat{f}^{F}-\hat{f}^{N}\right) \hat{u}^{\downarrow} \\
& -G^{\uparrow \downarrow} \hat{u}^{\dagger} \hat{f}^{N} \hat{u}^{\downarrow}-\left(G^{\uparrow \downarrow}\right)^{*} \hat{u}^{\downarrow} \hat{f}^{N} \hat{u}^{\dagger},
\end{aligned}
$$

where we have introduced the spin dependent conductances $G^{s}$

$$
G^{s}=\frac{e^{2}}{h}\left[M-\sum_{n m}\left|r_{s}^{\mathrm{nm}}\right|^{2}\right]=3 D \frac{e^{2}}{h} \sum_{n m}\left|t_{s}^{\mathrm{nm}}\right|^{2}
$$

and the mixing conductance

$$
G^{\uparrow \downarrow}=\frac{e^{2}}{h}\left[M-\sum_{n m} r_{\uparrow}^{\mathrm{nm}}\left(r_{\downarrow}^{\mathrm{nm}}\right)^{*}\right] .
$$

We thus see that the relation between the current through a contact and the distribution in the ferromagnetic reservoir and the normal metal node is determined by four conductances, the two real spin conductances $\left(G^{\dagger}, G^{\downarrow}\right)$ and the real and imaginary parts of the mixing conductance $G^{\Uparrow \downarrow}$. These contact-specific parameters can be obtained by microscopic theory or from experiments. The spin conductances $G^{\uparrow}$ and $G^{\downarrow}$ have been used in descriptions of spin transport for a long time [1]. The mixing conductance is a new concept which is relevant for transport between noncollinear ferromagnets. The mixing conductance rotates spins around the magnetization axis of the ferromagnet. Note that although the mixing conductance is a complex number, the $2 \times 2$ current in spin space is Hermitian and, consequently, the current and the spin current 
in Eq. (3) are real numbers. Generally we can show that $\operatorname{Re} G^{\dagger \downarrow} \geq\left(G^{\uparrow}+G^{\downarrow}\right) / 2$. Below, we present explicit results for the conductances when the contacts are in the diffuse, tunneling, and ballistic regimes.

For a diffuse contact, Eq. (3) can quite generally be found by the Green function technique developed in Ref. [11]. Here we use a much simpler approach based on the diffusion equation. On the normal metal side of the contact the boundary condition to the diffusion equation is set by the distribution matrix in the node $\hat{f}^{N}$. On the ferromagnet side of the contact the boundary condition is set by the equilibrium distribution function in the reservoir $f^{F} \hat{1}$. In a ferromagnetic metal, transport of spins noncollinear to the local magnetization leads to a relaxation of the spins since electrons with different spins are not coherent. This causes an additional resistance, which as other interface related excess resistances, is assumed to be small compared to the diffuse bulk resistance. Sufficiently far from the ferromagnet-normal metal interface the distribution function of the electronic states in the ferromagnet can always be represented by two components. Only the spin current parallel to the magnetization of the ferromagnet is conserved. We denote the cross section of the contact $A$, the length of the ferromagnetic part of the contact $L^{F}$, the length of the normal part of the contact $L^{N}$, the (spin dependent) resistivity in the ferromagnet $\rho^{F s}$, the resistivity in the normal metal $\rho^{N}$, so that the (spin dependent) conductance of the ferromagnetic part of the contact is $G^{D F s}=A /\left(\rho^{F s} L^{F}\right)$ and the conductance of the normal part of the contact is $G^{D N}=A /\left(\rho^{N} L^{N}\right)$. Solving the diffusion equation $\nabla^{2} \hat{f}=0$ on the normal and ferromagnetic side with the boundary conditions above, we find the current through a diffuse contact:

$$
\begin{aligned}
\hat{i}^{D}= & G^{D \dagger} \hat{u}^{\dagger}\left(\hat{f}^{F}-\hat{f}^{N}\right) \hat{u}^{\dagger}+G^{D \downarrow} \hat{u}^{\downarrow}\left(\hat{f}^{F}-\hat{f}^{N}\right) \hat{u}^{\downarrow} \\
& -G^{D N}\left(\hat{u}^{\dagger} \hat{f}^{N} \hat{u}^{\downarrow}+\hat{u}^{\downarrow} \hat{f}^{N} \hat{u}^{\dagger}\right),
\end{aligned}
$$

where the total spin dependent conductance is $1 / G^{D s}=$ $1 / G^{D F s}+1 / G^{D N}$. This result can be understood as a specific case of the generic Eq. (3) with $G^{\uparrow}=G^{D \uparrow}, G^{\downarrow}=$ $G^{D \downarrow}$, and $G^{\uparrow \downarrow}=G^{D N}$. The mixing conductance in the diffuse limit therefore depends on the conductance of the normal part of the contact only, which is a consequence of the relaxation of spins noncollinear to the magnetization direction in the ferromagnet.

For a ballistic contact, we use a simple semiclassical model proposed in Ref. [12]. In this model the channels are either completely reflected or transmitted, with $N^{\uparrow}$ and $N^{\downarrow}$ being the number of transmitted channels for different spin directions. Substituting this in (1) we find that the spin conductance $G^{B \uparrow}=\left(e^{2} / h\right) N^{\uparrow}, G^{B \downarrow}=\left(e^{2} / h\right) N^{\downarrow}$ and the mixed conductance is determined by the lowest number of reflected channels, $G^{B \uparrow \downarrow}=\max \left(G^{B \dagger}, G^{B \downarrow}\right)$ and is real.

For a tunneling contact we can expand Eq. (1) in terms of the small transmission. We find that $\operatorname{Re} G^{T \uparrow \downarrow}=$
$\left(G^{T \uparrow}+G^{T \downarrow}\right) / 2$, where $G^{T \uparrow}$ and $G^{T \downarrow}$ are the tunneling conductances. The imaginary part of $G^{T \uparrow \downarrow}$ can be shown to be of the same order of magnitude as $G^{T \uparrow}$ and $G^{T \downarrow}$ but it is not universal.

We will now illustrate the theory by computing the current through the three-terminal device shown in Fig. 2. A normal metal node $(N)$ is connected to three ferromagnetic reservoirs $(F 1, F 2$, and $F 3)$ by arbitrary contacts parametrized by our spin conductances. A source-drain bias voltage $V$ applied between reservoirs 1 and 2 causes an electric current $I$ between the same reservoirs. The charge flow into reservoir 3 is adjusted to zero by the chemical potential $\mu_{3}$. Still, the magnetization direction $\mathbf{m}_{3}$ influences the current between reservoir 1 and 2 . We assume that spin relaxation in the normal node can be disregarded so that the right-hand side of (2) is set to zero. Furthermore, we assume that the voltage bias $V$ is sufficiently small so that the energy dependence of the transmission (reflection) coefficients can be disregarded. To further simplify the discussions the contacts 1 and 2 are taken to be identical, $G_{1}^{\uparrow}=G_{2}^{\uparrow} \equiv G^{\uparrow}, G_{1}^{\downarrow}=G_{2}^{\downarrow} \equiv G^{\downarrow}$, and $G_{1}^{\Uparrow \downarrow}=G_{2}^{\Uparrow \downarrow} \equiv G^{\uparrow}$. Contact 3 is characterized by the conductances $G_{3}^{\uparrow}, G_{3}^{\downarrow}$, and $G_{3}^{\Uparrow \downarrow}$. We find the distribution in the normal node by solving the four linear Eqs. (2). The current through the contact between reservoir 1 (2) and the node is obtained by inserting the resulting distribution for the normal node into Eq. (3).

When the magnetizations in reservoirs 1 and 2 are parallel there is no spin accumulation since contacts 1 and 2 are symmetric and, consequently, ferromagnet 3 does not affect the transport properties. The current is then simply a result of two total conductances $G=G^{\uparrow}+G^{\downarrow}$ in series, $I=G V / 2$. The influence of ferromagnet 3 is strongest when there is a significant spin accumulation in the normal metal node, and in the following the magnetizations of the source and drain reservoirs are

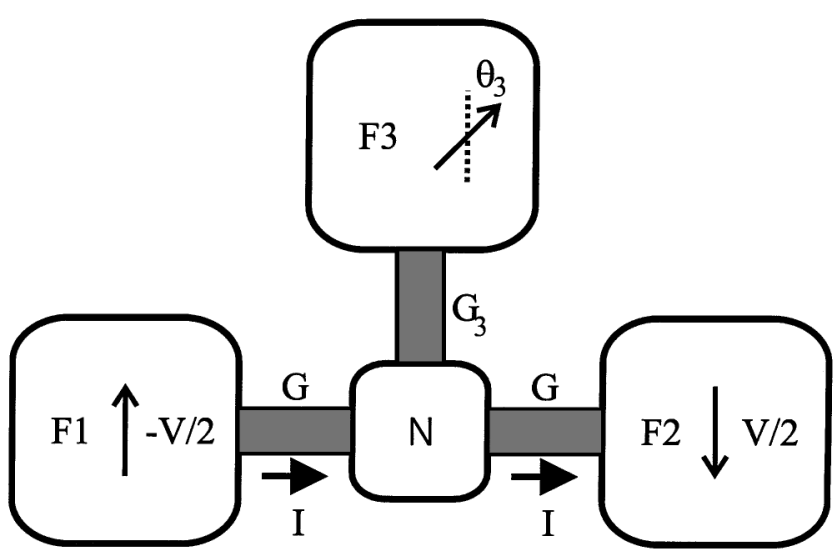

FIG. 2. The three-terminal device where a normal metal node is connected to ferromagnetic reservoirs. An applied bias causes a source-drain current between $F 1$ and $F 2$. The charge current into $F 3$ is adjusted to vanish by $\mu_{3}$. The magnetization direction of ferromagnet F3 controls the current. 


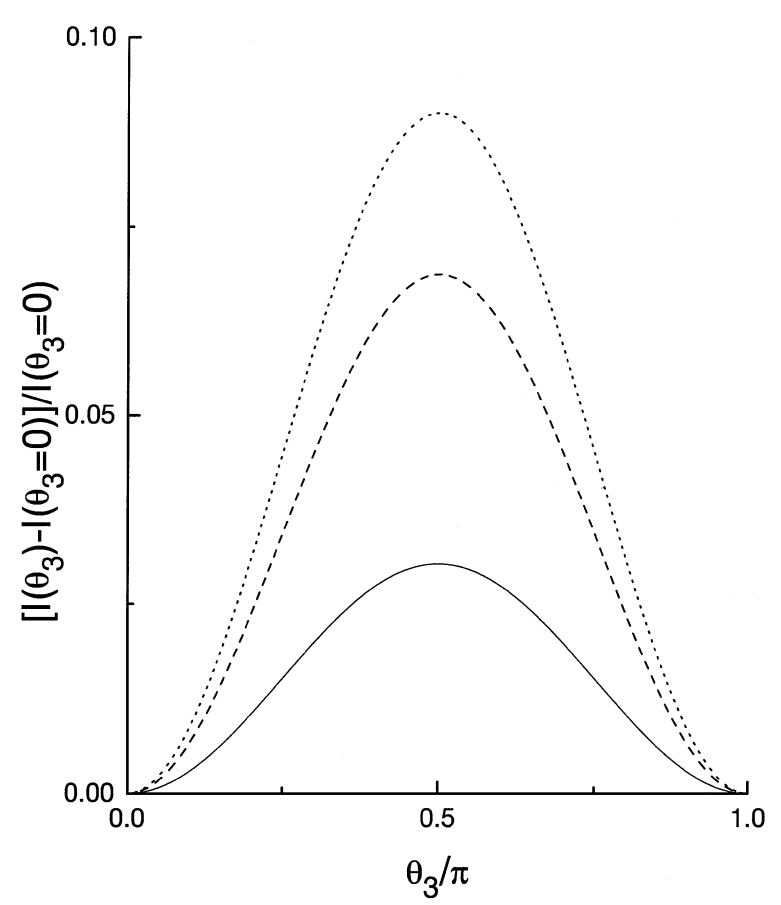

FIG. 3. The current $\operatorname{Tr}\left(i_{1}\right)=-\operatorname{Tr}\left(i_{2}\right)$ in the three terminal device as a function of the magnetization direction $\theta_{3}$. The straight, dashed, and dotted lines correspond to the relative mixing conductance $\operatorname{Re} \eta_{2}=2,5$, and 10, respectively. The other parameters were set to $P=3 D 0.4, P_{3}=0.1, G=G_{3}, \operatorname{Re} \eta_{3}=1.0$, $\operatorname{Im} \eta=0=\operatorname{Im} \eta_{3}$.

antiparallel, $\mathbf{m}_{1} \cdot \mathbf{m}_{2}=-1$. We denote the relative angle between the magnetizations in reservoirs 3 and 1 (reservoir 2) $\theta_{3}\left(\pi-\theta_{3}\right)$. The current is an even function of $\theta_{3}$ and symmetric with respect to $\theta_{3} \rightarrow \pi-\theta_{3}$ as a result of the symmetry of the device, e.g., the current when the magnetizations in reservoirs 1 and 3 are parallel equals the current when the magnetizations in reservoirs 1 and 3 are antiparallel. Because of the finite mixing conductance at noncollinear magnetization the third contact acts as a drain for the spin accumulation in the node, thus allowing a larger charge current between reservoirs 1 and 2 . The relative increase of the current due to the reduced spin accumulation $\Delta_{3}\left(\theta_{3}\right)=\left[I\left(\theta_{3}\right)-I\left(\theta_{3}=0\right)\right] / I\left(\theta_{3}=0\right)$, is plotted in Fig. 3 as a function of $\theta_{3}$. The maximum of $\Delta_{3}$ is achieved at $\theta_{3}=\pi / 2\left(\theta_{3}=3 \pi / 2\right)$ and equals $\left(\operatorname{Im} G_{3}^{\uparrow \downarrow}=0\right)$

$$
\Delta_{3}=P^{2} \frac{2 G G_{3}}{2 G+G_{3} \eta_{3}} \frac{\eta_{3}-1+P_{3}^{2}}{2 G\left(1-P^{2}\right)+G_{3}\left(1-P_{3}^{2}\right)}
$$

introducing the total conductance of the contact $G_{i}=G_{i}^{\uparrow}+G_{i}^{\downarrow}$, the polarization of the contact $P_{i}=\left(G_{i}^{\uparrow}-G_{i}^{\downarrow}\right) /\left(G_{i}^{\uparrow}+G_{i}^{\downarrow}\right)$ and the relative mixing conductance $\eta=2 G_{i}^{\uparrow \downarrow} /\left(G_{i}^{\uparrow}+G_{i}^{\downarrow}\right)$. The influence of the direction of the magnetization of reservoir 3 increases with increasing polarization $P$ and increasing relative mixing conductance $\eta_{3}$ and reaches its maximum when the total conductances are of the same order $G_{3} \sim G$. Note that the physics of this three-terminal device is very different from that of Johnson's spin transistor [5]; the latter operates with collinear magnetizations of two ferromagnetic contacts, whereas the third may be normal.

In conclusion, we have proposed a finite-element transport theory for spin transport in mesoscopic systems. In the presence of ferromagnetic order a contact can be described by four conductance parameters which we obtained explicitly for diffuse, ballistic, and tunnel contacts. We have applied the theory to a novel three-terminal device with arbitrary contacts.

This work is part of the research program for the "Stichting voor Fundamenteel Onderzoek der Materie" (FOM), which is financially supported by the "Nederlandse Organisatie voor Wetenschappelijk Onderzoek" (NWO). We acknowledge benefits from the TMR Research Network on "Interface Magnetism" under Contract No. FMRX-CT960089 (DG12-MIHT) and support from the NEDO joint research program (NTDP-98). We acknowledge stimulating discussions with Wolfgang Belzig, Daniel Huertas Hernando, and Junichiro Inoue.

*Also at Philips Research Laboratories, Professor Holstlaan 4, 5656 AA Eindhoven, The Netherlands.

Present address: Harvard University, Lyman Laboratory of Physics, Cambridge, MA 02138.

[1] P. M. Levy, Solid State Phys. 47, 367 (1994); M. A. M. Gijs and G. E. W. Bauer, Adv. Phys. 46, 285 (1997).

[2] A. Moreo, S. Yunoki, and E. Dagotto, Science 283, 2034 (1999).

[3] J. S. Moodera and L. R. Kinder, J. Appl. Phys. 79, 4724 (1996).

[4] J. C. Slonczewski, Phys. Rev. B 39, 6995 (1989); T. Valet and A. Fert, Phys Rev. B 48, 7099 (1993); V. V. Ustinov and E. A. Kravtsov, J. Phys. Condens. Matter 7, 3471 (1995); H. E. Camblong, P. M. Levy, and S. Zhang, Phys. Rev. B 51, 16052 (1995).

[5] M. Johnson and R. H. Silsbee, Phys. Rev. Lett. 55, 1790 (1985); M. Johnson, Phys. Rev. Lett. 70, 2142 (1993); M. Johnson, Science 260, 320 (1993).

[6] K. Ono, H. Shimada, S. Kobayashi, and Y. Ootuka, J. Phys. Soc. Jpn. 65, 3449 (1996).

[7] A. Brataas, Yu. V. Nazarov, J. Inoue, and G. E. W. Bauer, Phys. Rev. B. 59, 93 (1999); Eur. Phys. J. B 9, 421 (1999).

[8] Yu. V. Nazarov, Phys. Rev. Lett. 73, 1420 (1994).

[9] M. Büttiker, Phys. Rev. Lett. 57, 1761 (1986).

[10] D. H. Hernando, Yu. V. Nazarov, A. Brataas, and G. E. W. Bauer (unpublished).

[11] Yu. V. Nazarov, Phys. Rev. Lett. 73, 134 (1994).

[12] G. E. W. Bauer, Phys. Rev. Lett. 69, 1676 (1992); M. J. M. de Jong and C. W. J. Beenakker, Phys. Rev. Lett. 74, 1657 (1995). 